\title{
Effects of mulligan concept in classical ballet dancers with pain in the lower limbs
}

\author{
Letícia Lopes Aguiar, Vanessa Assunção Dos Santos Araújo, Laiana Sepúlveda De Andrade Mesquita.
}

Universidade Estadual do Piauí (PI), Brazil.

\begin{abstract}
Background: The Mulligan concept is a manual therapy technique that addresses the restoration of inadequate arthrokinematic processes and osteokinematic movements, resulting in reduced muscle tension, postural failures and pain relief. Objective: Analyze the effectiveness of the Mulligan concept in reducing lower limb pain processes in classical Ballet dancers. Methods: Longitudinal study, approved by the Ethics and Research Committee of the Universidade Estadual do Piauí with number 2064503, composed of 4 classical Ballet dancers. An evaluation of the pain threshold was performed using a digital algometer (Wagner Instruments) with a capacity of ten kilograms-force (Kgf) in the Ballet dancers who presented painful symptoms in the knee. The pain threshold was verified before and after the intervention with the Mulligan concept at the following points: patellar ligament (PL), medial collateral ligament (MCL) and lateral collateral ligament (LCL). The Mulligan concept was performed actively, without pain, with adjustments by the therapist and was maintained at the end of the knee range for 10 seconds. This protocol was repeated 3 times in the first visit, 10 times in the second, 2 repetitions of 10 slides in the third and in the subsequent visits there were 3 repetitions of 10 slides. Results: An increase in the mean of the pain threshold in the patellar, medial collateral and lateral collateral ligaments was observed in all the research participants, when comparing the initial values of the first day and the final values of the 12th session of Mulligan. The lateral collateral ligament presented the most significant results $(p=0.02)$. Statistical analysis was performed using the BioEstat 5.0 software. Shapiro-Wilk tests to assess the normality of the variables and the $t$-student test were applied, being considered statistically significant $(p<0.05)$. Conclusion: The lateral collateral ligament showed a statistically significant immediate and lasting improvement in the pain threshold of the Ballet dancers with the therapeutic intervention of the Mulligan concept.
\end{abstract}

Keywords: Dance; Musculoskeletal Manipulation; Musculoskeletal System.

\section{BACKGROUND}

Classical Ballet emerged in the Modern Age with the creation of the "Royal Dance Academy", founded by Louis XIV in 1661, due to the improvement of several dances already existing at the time ${ }^{(1,2)}$. During its development process, it stopped being instinctive and recreational, evolving to an artistic dance with complex body and choreographic compositions $^{(2)}$.

The classical ballet technique is based on "turnout" or rotation of the legs. Therefore, the movements of the ballet must be performed in an outward position. The ideal would be $180^{\circ}$ external rotation starting at the hips, resulting as a consequence that the feet are easily placed in a $180^{\circ}$ position on the floor. Many students are unable to reach the ideal position in the execution of the movements because of limitations in the rotation of the hip, knee and the difficulty of putting their feet in the correct position. A dancer can use pronation or roll inside the median arch and ankle, placing increased torque on the median ankle, tibia and knee. This forced convergence can lead to foot, ankle and knee injuries, and also cause tension in structures around the ankle and knee ${ }^{(3,4)}$. Ballet dancers have a high level of postural control and stability due to the sensitivity acquired by their proprioceptors in the occurrence of constant balance disturbances suffered during the performance of the dance movements ${ }^{(5)}$. However, repetitive movements and non-physiological body alignment, represented by lateral rotation of the hip, knee and ankle, also known as the "en dehors" position in classical ballet, and support at the ends of the metatarsals, known as the "tip or half position tip "of the feet, lead to maximum plantar flexion of the ankle, hip hyperextension and bodily misalignment, thus becoming the main risk factors of the profession, generating severe pain and musculoskeletal injuries ${ }^{(6,7)}$.

The improper execution of the technique, associated with biomechanical imbalances, results in the primary factor of chronic musculoskeletal injuries of the lower limbs ${ }^{(8,9)}$. Thus, as a result of the compensatory mechanism, there is an overload of the joint, resulting in knee pain, especially in the tibiofemoral and patellofemoral joints ${ }^{(8,10)}$. Thus, the prevalence of knee injuries is high, representing approximately $20 \%$ of all injuries in the lower limbs $^{(11,12)}$ and with atraumatic and chronic features. In the musculoskeletal treatment of joint disorders, 
the physiotherapist can use manual therapy as a solid treatment base ${ }^{(13-15)}$. One of these manual therapy techniques includes Mobilization with Movement (MWM), a type of joint mobilization developed by Brian Mulligan ${ }^{(16,17)}$. Mulligan's original theory for the effectiveness of a MWM is based on the concept related to a joint positional failure, which occurs due to injury and can lead to a change in joint alignment and, consequently, in its biomechanics, resulting in symptoms such as pain, joint stiffness or weakness ${ }^{(16)}$.

The Mulligan MWM concept is a manual therapy technique that was designed to address restoration defects in arthrokinematic processes and osteokinematic movements ${ }^{(18)}$. Mulligan raised the hypothesis of a joint position failure that must be identified and corrected. When MWM ceases pain, it restores function and provides a long-lasting therapeutic effect ${ }^{(19)}$. Thus, it is a concept that may be appropriate for pain relief, reduction of muscle tension and postural failures.

Finally, there is a notable lack of relevant literature and evidence for the analysis and construction of the present study. Thus, it aims to analyze the effectiveness of the Mulligan concept in reducing pain symptoms in the lower limbs of classical Ballet dancers.

\section{METHODS}

It is a longitudinal, analytical and quantitative clinical trial conducted with 4 classical Ballet dancers in a ballet school in Teresina (PI) which is a reference in the teaching and training of classical Ballet dancers.

Classical Ballet dancers aged between 12 and 30 years were included in the study: who present painful symptoms in their knees; who have at least 2 years of practice of this dance; and who agreed to sign the Informed Consent Form, according to Resolution $\mathrm{n}^{\circ} 466 / 2012$, signed by the participant, and the Term of Assent, signed by the person responsible for participants under the age of 18 years. Ballet dancers who dropped out of the study and those who missed more than two consecutive sessions were excluded.

After approval by the Research Ethics Committee of the State University Piauí according to the № 2064503 of 05/15/2017 and authorization from the co-participant institution, classical Ballet dancers were invited to participate in the study and on this occasion the consent form was read and explained and later delivered and signed by those who agreed to participate in the research.

Fifteen Ballet dancers were invited to participate in the research, with an average age range of 21.3 years, however after applying the non-inclusion criteria as well as the exclusion criteria, only 12 remained for therapeutic intervention. Of these 12 Ballet dancers, only 4 completed the treatment, as the rest had an inadequate frequency resulting in an excessive amount of absences that could interfere with the expected result.

\section{Evaluation protocol}

Data collection was performed by means of a global assessment of the classical Ballet dancers included using the following parameters:

1. Evaluation Form and Questionnaire: The aspects used were related to identification data, classical ballet, pain and injuries.

2. Visual Analog Scale (VAS): Assesses and assists in measuring the intensity of pain in the subject using a straight line, indicating at one end the marking "no pain", and at the other, "worst pain imaginable".

3. Pain assessment by algometer: The assessment of the pain threshold is performed by means of a digital algometer (Wagner Instruments) with a capacity of ten kilograms-force (Kgf) and compression deformation of 10 centimeters $(\mathrm{cm})$. This quantifies the sensitization of the subcutaneous tissue, by means of a device, which positioned over the painful site causes a pressure that is slowly increased. The amount of pressure is usually recorded as a pain threshold, which is the level that subjects report feeling discomfort.

After the consent of the participants who showed interest in the study and the data collection, there was an initial evaluation for screening the subjects who met the inclusion criteria. Participants were assessed at the beginning, right after the first appointment and after 4 weeks. Three visits per week were performed, according to the availability of each patient's schedule, totaling 12 sessions, the last evaluation being one week after the last appointment to assess the late effect of the Mulligan concept. There was no specific length of time for each service, as throughout the intervention there was an increase in repetition of the accessory slip movements. The VAS collected in the sample was standardized in the 1st, 5th and 12th visits to monitor the pain intensity during the treatment protocol.

To perform the MWM technique, it was necessary to identify the movement in which the dancer felt pain in the knee joint. From the execution of the movement that caused pain, a maintained accessory slip was applied, in order to correct the positional failure of the joint. The movement, which normally caused pain, was performed either actively or passively without pain and held at the end of the range for 10 seconds. This procedure was repeated 
3 times in the first visit, in the second 10 times, 2 repetitions of 10 slides in the third and in the subsequent visits there were 3 repetitions of 10 slides.

An essential aspect of MWM is pain, which must always be reduced and/or eliminated during application $^{(16,20)}$. Thus, the Ballet dancers were instructed to report any discomfort during the slides, to change the direction of the accessory slip movements on the knee if necessary, or even interrupt the service.

After each MWM service, the ability of the dancer to perform the same movement that previously caused pain was evaluated, without the aid of manual application of the maintained accessory slip or the ability of the dancer in a functional task.

\section{Statistical analysis}

Data processing and analysis were performed using the BioEstat 5.0 software. First, a descriptive analysis of the variables was performed to verify the mean and standard deviation. As the variables followed the normal pattern, Student's t test for paired samples was performed, considering statistically significant values of $p<0.05$.

\section{RESULTS}

Thus, data were collected regarding the pain threshold in the patellar (PL), medial collateral $(\mathrm{MCL})$ and lateral collateral (LCL) ligaments in the knee joint. Graphs 1, 2 and 3 below show the pain threshold collected by the algometer from the 1st to the 12th attendance. The VAS collected in the sample was standardized in the 1st, 5th and 12th visits. From what is shown in Table 1 below, notice that the value was found with the course of the physiotherapy intervention, demonstrating the evolution in the pain of Ballet dancers.

The four classical Ballet dancers had a greater painful complaint in the left lower limb when compared to the right, corroborating with the data observed in the individual assessment sheets of each participant, in which they reported their dominance in the classes corresponding to the left laterality.

This means that, according to the participants' perception, the left side presents a better execution and performance of the exercises when compared to the right side. Thus, the left lower limb has a greater stress overload of movements, causing more frequent pain in that knee. The Mulligan concept movements used in the volunteers were anterior, posterior, medial, lateral and rotational sliding, according to the most comfortable position for them during the movement that caused pain.

Descriptive statistics were performed to identify the mean and standard deviation of the ligament variables analyzed in this study. In the patellar ligament, medial collateral and lateral collateral presented, respectively, the value of $p=$ $0.08 ; p=0.23$; and $p=0.02$.

Table 1. Evolution of the pain threshold measured by VAS in classical Ballet dancers.

\begin{tabular}{ccccc}
\hline & Patient 1 & Patient 2 & Patient 3 & Patient 4 \\
\hline $1^{\circ}$ & 7 & 8 & 6 & 8 \\
VAS & & & & \\
$2^{\circ}$ & 5 & 5 & 5 & 6 \\
VAS & & & & \\
$3^{\circ}$ & 4 & 3 & 5 & 4 \\
VAS & & & &
\end{tabular}

${ }^{\bar{N}}$ Note: VAS - Visual Analogue Scale.

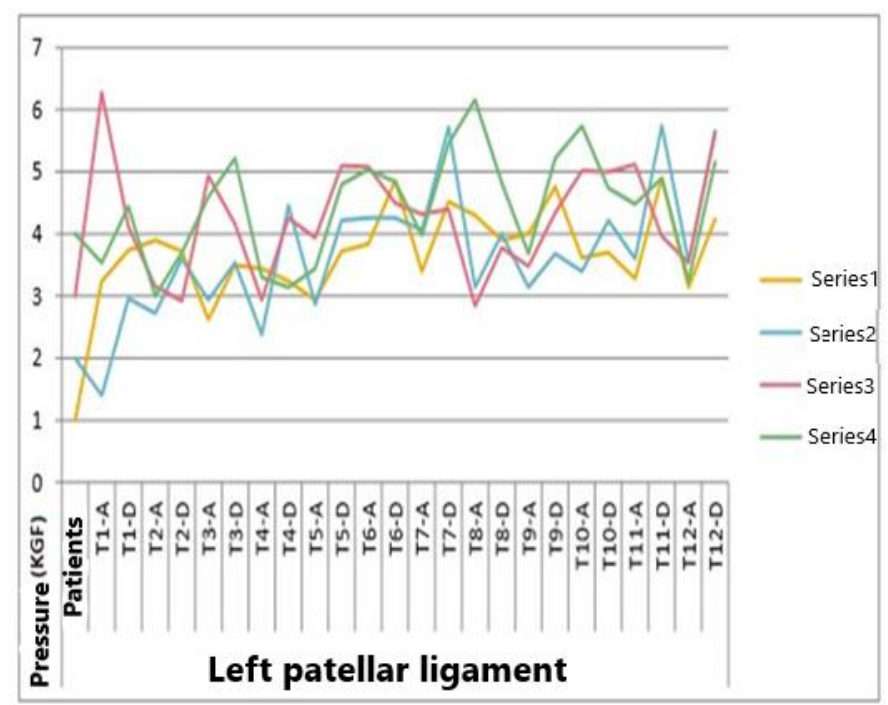

Figure 1. Evolution of the pain threshold measured by the algometer in the left patellar ligament in classical Ballet dancers.

"Note: T1-A: 1st session before applying the Mulligan; T1-D: 1st session after the application of Mulligan; Series: patient; Pressure in $\mathrm{kgF}$.

In the left patellar ligament, it is possible to observe that the final pressure values caused by the algometer were higher in the 12th Mulligan treatment when compared to the 1st in general and individually in all patients, in which initially the values varied from 1 to $4 \mathrm{kgF}$ and at the end sessions ranged from 2 to $6 \mathrm{kgF}$. 


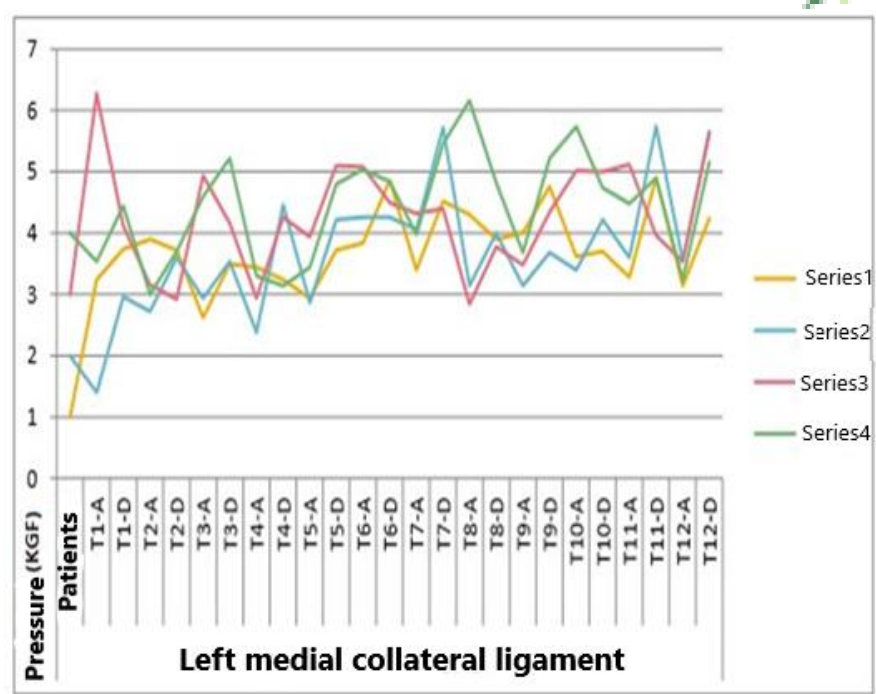

Figure 2. Evolution of the pain threshold measured by the algometer in the left medial collateral ligament in classical ballerinas.

"Note: T1-A: 1st session before applying the Mulligan; T1-D: 1st session after the application of Mulligan; Series: patient; Pressure in $\mathrm{kgF}$.

In the left medial collateral ligament, it is observed that the final pressure values caused by the algometer were higher in the 12th Mulligan treatment when compared to the 1st in general and individually in all patients, in which initially the values varied from 1 to $4 \mathrm{kgF}$ and at the end of the sessions they ranged from 4 to $6 \mathrm{kgF}$.

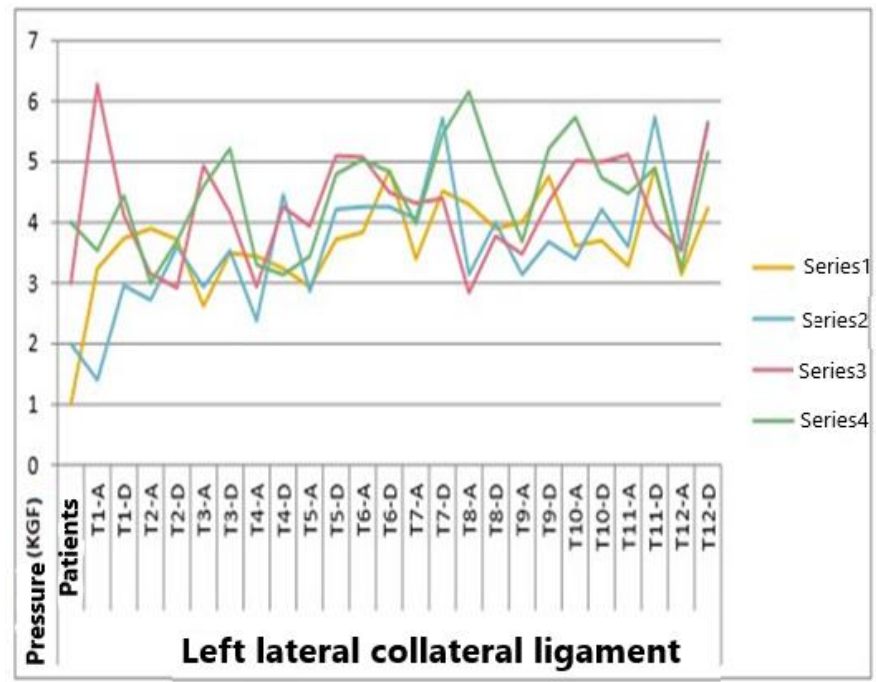

Figure 3. Evolution of the pain threshold measured by the algometer in the left lateral collateral ligament in classical ballerinas.

"Note: T1-A: 1st session before applying the Mulligan; T1-D: 1st session after the application of Mulligan; Series: patient; Pressure in $\mathrm{kgF}$.

In the left lateral collateral ligament, it is observed that the final pressure values caused by the algometer were higher in the 12th Mulligan treatment when compared to the 1st in general and individually in all patients, in which initially the values varied from 1 to $4 \mathrm{kgF}$ and at the end of the sessions they ranged from 5 to almost $10 \mathrm{kgF}$. This demonstrates that this ligament showed a statistically significant improvement, in relation to the other results found, due to the excessive use of external rotation of the lower limbs, evidenced in classical Ballet dancers.

\section{DISCUSSION}

The main tendon injury in Ballet dancers is knee tendinopathies, as this joint receives an intense overload during some specific jumping movements in this dance, causing biomechanical stress in the structures of that joint ${ }^{(21)}$.

The Mulligan concept has mechanical and physiological properties that cause mobilization of joint correction, repositioning it in the appropriate place, restoring painless function and providing lasting benefits ${ }^{(17)}$.

A randomized controlled study in patients with shoulder impact syndrome, with a sample of 42 women, showed as results a greater reduction in pain intensity during shoulder flexion, greater range of motion of shoulder flexion without pain and maximum external rotation of shoulder in patients who received Mobilization with Movement (MWM) when compared to patients who participated in the control group ${ }^{(22)}$. These observed benefits corroborate those reported by the Ballet dancers participating in the present study.

The experimental study with a sample of 34 patients, a significantly greater improvement in the maximum grip strength and the average VAS in patients with lateral epicondylitis in the experimental group, which received the combination of Mulligan technique with traditional treatment, than in the control group that received only traditional treatment ${ }^{(23)}$. They also reported an improvement in their ability to perform daily functional activities.

Another study reported a treatment performed on a 14-year-old dancer with a foot contusion for 3 weeks, in which pain made plantar flexion impossible, the movement known as "tips" in classical ballet ${ }^{(17)}$. The application of sustained downward mobilization at the base of the second metatarsal with the third was used, allowing painless movement and return to normal activities after a few sessions.

It was also discovered that MWM provided a statistically significant improvement in functional performance, pain reduction and induced a positive gain in muscle strength in patients with symptomatic lateral epicondylitis, when comparing treatment in 3 intervention groups: Mulligan, wrist joint manipulations and the group control(24). 
As well as the benefits reported by these studies, the Ballet dancers in this research reported a better performance at the end of the physiotherapy treatment in classes and during rehearsal periods, reduced intensity and frequency of pain, and decreased need to use orthoses, such as the tensor, in the knee joint.

\section{CONCLUSION}

The lateral collateral ligament (LCL) presents greater stress and overload in the movements of classical Ballet due to the excessive external rotation of the lower limbs characteristic of Ballet dancers (en dehors). This ligament showed a statistically significant immediate and lasting improvement in the pain threshold of the Ballet dancers with the therapeutic intervention of the Mulligan concept.

Despite the indisputable importance of the Mulligan concept in joint readjustment to restore physiological and painless function, and the consequent benefits of this adjustment, it is necessary to produce more scientific studies that show this concept, specifically in the knee joint, and that can characterize better the physiotherapeutic conduct that can be used in the population of classical Ballet dancers. Thus, it is possible to develop means of prevention and appropriate physical therapy accompaniments in schools, companies, gyms and dance centers.

\begin{abstract}
Authors' contributions: LLA academic researcher, responsible for the execution of the study, data collection, tabulation of the collected data and scientific writing of the research. VASA collaborating researcher, responsible for data collection and critical review of the research. LSAM guiding researcher and supervisor of the study, responsible for the design, guidelines, statistical analysis and critical review of the research. All authors read and approved the final version of the study.
\end{abstract}

Financial support: The authors declare that there is nonfinancial competing interests

Conflict of interest: The authors declare that there was no conflict of interests.

\section{REFERENCES}

1. Haas NA, Garcia AD, Bertoletti J. Imagem Corporal e Bailarinas Profissionais. Rev Bras Med Esporte. 2010;16(3):182-185.

2. Simas JPN, Guimarães ACA. Ballet clássico e transtornos alimentares. $\mathrm{R}$ Educação Física/UEM. 2002;13(2):119-126.

3. Howse J. Dance technique \& injury prevention. 3rd ed. New York: Routledge; 2000.

4. Macintyre J, Joy E. Foot and ankle injuries in dance. Clin Sports Med. 2000;19(2):351-68.
5. Kiefer AW, Riley MA, Shockley K, Sitton CA, Hewett TE, Sebree SC, et al. Multi-segmental postural coordination in professional ballet dancers. Gait \& Posture. 2011;34:76-80.

6. Hillier JC, Peace K, Hulme A, Healy JC. Pictorial review: MRI features of foot and ankle injuries in ballet dancers. Br J Radiol. 2004;77:532-537.

7. Lin CF, Lee IJ, Liao JH, Wu HW, Su FC. Comparison of Postural Stability Between Injured and Uninjured Ballet Dancers. Am J Sports Med. 2011;39:1324-1331.

8. Steimberg N, Siev-Ner I, Peleg S, Dar G, Masharawi $Y$, Zeev $A$, et al. Joint range of motion and patellofemoral pain in dancers. Int $\mathrm{J}$ Sports Med. 2012;33(7):561-566.

9. Thomas H, Tarr J. Dancers' perceptions of pain and injury: positive and negative effects. J Dance Med Sci. 2009;13(2):51-9.

10. Solomon RL, Solomon J, Minton SC. Preventing dance injuries. 2th ed. Champaign: Human Kinematics; 2005.

11. Gamboa JM, Maring J, Roberts LA, Forgus A. Injury patterns in elite preprofessional ballet dancers and the utility of screening programs to identify risk characteristics. J Orthop Sports Phys Ther. 2008;38(3):126-136.

12. Stretanski MF, Weber GJ. Medical and reabilitation issues in classical ballet. Am J Phys Med Rehabil. 2002;81(5):383-91.

13. Hernandez-Molina G, Reichenbach S, Zhang B, Lavalley M, Felson D. Effect of Therapeutic Exercise for Hip Osteoarthritis Pain: Results of a Meta-Analysis. Arthritis \& Rheumatism; 2008. p. 1221-1228.

14. Fransen M, McConnell S, Hernandez-Molina G, Reichenbach S. Exercise for osteoarthritis of the hip (review). Cochrane; 2009. p. 1-24.

15. French HP, Brennan A, White B, Cusack T.Manual therapy for osteoarthritis of the hip or knee e A systematic review. Elsevier, 2011.p.109-117.

16. Mulligan B. Manual therapy: "NAGS", "SNAGS", "MWMs" ect.5th ed. Wellington: Plane View Services Ltd, 2004.

17. Mulligan B. Mulligan Concept.2007.

18. Folk B, Crowell R, Mulligan B. Introduction to the Mulligan Concept Workbook. East Hampstead: $\mathrm{NH}$ : Northeast Seminars; 2012.

19. Mulligan BR. Manual Therapy: "NAGS," SNAGS," MWMS," etc. 6th ed. Wellington, New Zealand: Plane View Services LTD; 2010.

20. Hing W. Mulligan's mobilization with movement: a review of the tenets and presciption of MWM's. NZ journal of physiotherapy. 2008;144-164.

21. Couto AGA, Pedroni CR. Relação entre postura, queixa dolorosa e lesão em bailarinas 
clássicas. Revista terapia manual. 2013:228233.

22. Delgado-GilJ, Pado-Robles E, Rodrigues-deSouza DP, Cleland JÁ, Fernández-de-las-Peñas $C$, Albuquerque-Sendin F. Effects of mobilization with movement on pain and range of motion in patients with unilateral shoulder impingement syndrome: a randomized controlled trial. Journal of manipulative and physiological therapeutics. 2015;38(4):245-252.

23. Amro A, Diener I, Bdair WO, Hameda IM, Shalabi Al, Ilyyan DI. The effects of Mulligan mobilisation with movement and taping techniques on pain, grip strength, and function in patients with lateral epicondylitis. Hong Kong Physiotherapy Journal. 2010;28(1):19-23.

24. Geetu M, Deepak G. Effectiveness of movement with mobilization compared with manipulation of wrist in case of lateral epicondylitis. Indian Journal of Physiotherapy and Occupational Therapy. 2008;2(1):16-25. 